\title{
Toxinas e perfil protéico de amostras de Actinobacillus suis provenientes de plantéis suínos norte-americanos
}

\author{
Toxins and proteic profile of Actinobacillus suis samples from North American hog herds
}

\section{Rafael Silveira Carreon ${ }^{\mathrm{I}}$ Simone Rodrigues Oliveira ${ }^{\mathrm{I}}{ }^{*}$ Rone Cardoso $^{\mathrm{I}}$ João Helder Frederico de Faria Naves $^{\mathrm{I}}$ John Tomaszewski ${ }^{\mathrm{II}}$ Anna Monteiro Correia Lima Ribeiro ${ }^{\mathrm{I}}$}

\section{RESUMO}

\begin{abstract}
Actinobacillus suis (A.suis) surgiu como uma grande ameaça aos plantéis suínos norte-americanos. Os sinais clínicos e as lesões são particularmente variáveis e podem lembrar aquelas causadas por outros organismos, como o Actinobacillus pleuropneumoniae (App), podendo ter como causa a similaridade na produção das toxinas ApxI e ApxII. Os objetivos do estudo foram confirmar a produção das toxinas ApxI e ApxII, investigar a produção de toxina geneticamente semelhante à Apx III e analisar as proteínas totais, verificando se existe similaridade entre os isolados provenientes de diferentes plantéis de suínos norte-americanos. Neste estudo, todas as cepas de A. suis foram positivas para os genes codificadores das toxinas ApxI e ApxII, usando o método de reação em cadeia de polimerase - multiplex (PCR-multiplex); e as proteínas totais de 70 amostras de A. suis, oriundos de diferentes plantéis suinos norte-americanos, foram analisadas por meio de eletroforese em gel de poliacrilaminda desnaturante (SDS-PAGE) e foram idênticas. A similaridade eletroforética observada entre as proteínas totais das bactérias analisadas indica a possibilidade de haver uma proteção cruzada a partir de uma provável vacina universal desenvolvida com esses antígenos para A. suis.
\end{abstract}

Palavras-chave: Actinobacilose suína, ApxI, ApxII, pleuropneumonia suína, PCR-Multiplex.

\section{ABSTRACT}

Actinobacillus suis (A. suis) has arisen as a great threat to the North American hog herds. The clinical symptoms and lesions are particularly variable and may resemble the same caused by other pathogenic organisms, such as Actinobacillus pleuropneumoniae (App), which can similarly lead to the production of the toxins ApxI and ApxII. This study aimed to confirm the production of the toxins ApxI and ApxII, as well as, to investigate the production of toxins that are genetically similar to ApxIII, and analyze total protein to verify whether there is any similarity among the isolated samples obtained from different North American hog herds. In this study, all the strains of $\boldsymbol{A}$. suis were positive for the genes that codify the toxins ApxI and ApxII using the multiplex polymerase chain reaction (PCR-multiplex) method; and total protein from 70 samples of $\boldsymbol{A}$. suis, obtained from different North American hog herds, were analyzed through denaturing polyacrylamide gel electrophoresis (SDS-PAGE), and were identical. The electrophoretic similarity observed among total protein of the analyzed bacteria indicates that there is the possibility of existing a cross protection in case of developing a probable universal vaccine with the antigens of $\boldsymbol{A}$. suis.

Key words: swine actinobacilus, ApxI, ApxII, swine pleuropneumonia, PCR-Multiplex.

\section{INTRODUÇÃO}

Actinobacillus suis (A.suis) é um importante patógeno oportunista que pode causar doença em suínos de todas as idades (OJHA et al., 2010). O microrganismo possui componentes semelhantes aos de outros Gram negativos, como os lipopolissacarideos (LPS) e poros constituintes de toxinas (SLAVIC et al., 2000). As toxinas hemolíticas/ citotóxicas produzidas pelo $A$. suis são muito semelhantes geneticamente às toxinas do

'Laboratório de Doenças Infectocontagiosas, Faculdade de Medicina Veterinária, Universidade Federal de Uberlândia (UFU), Uberlândia, MG, Brasil.

IVeterinary Diagnostic Laboratory College of Veterinary Medicine University of Minnesota, 1333 Gortner Avenue St. Paul, MN, USA. 55108-1098. E-mail: oliv0107@umn.edu. *Autor para correspondência. 
Actinobacillus pleuropneumoniae (App), ApxI e ApxII (KAMP et al., 1994). A similaridade na produção de toxinas pode ser o fator determinante nas semelhanças observadas entre os sinais clínicos de infecções por A. suis e infecções por App.

As toxinas ApxI e ApxII encontradas em espécies consideradas menos virulentas como o A. suis foram denominadas de ApxIA var suis e ApxIIA var suis (BURROWS et al., 1992; OSTAAIJEN et al., 1997). Vários testes feitos por meio de reação em cadeia de polimerase (PCR), que são baseados nos genes que codificam as toxinas Apx, foram desenvolvidos para o diagnóstico da pleuropneumonia suína (COLLARES, 2000; GRAM et al.,2000). Entretanto, a homologia com os genes das toxinas ApxI varsuis e ApxII var suis de $\boldsymbol{A}$. suis impede a aplicação desses genes como alvo para o diagnóstico do agente (OSTAAIJEN et al., 1997; SCHALLER et al., 2000).

O diagnósticoé tradicionalmente realizado por meio de isolamento bacteriano, e a identificação da espécie por meio de testes bioquímicos (OLIVEIRA, 2007). Atualmente, não existem vacinas comerciais disponíveis para o controle de $\boldsymbol{A}$. suis, e a maioria dos veterinários dependem de vacinas autógenas e antibióticos para controlar e prevenir mortalidade (GEIGER et al., 2008).

Devido à necessidade da criação de uma vacina universal que tenha como característica a similaridade entre o isolado usado na produção desta e o restante de $\boldsymbol{A}$. suis presente nos plantéis, o estudo teve como objetivos confirmar a produção das toxinas ApxI e ApxII, investigar a produção de toxina geneticamente semelhante à ApxIII pelo A. suis e analisar os perfis proteicos, verificando se existe similaridade entre os isolados provenientes de diferentes plantéis de suínos norte-americanos.

\section{MATERIAL E MÉTODOS}

Amostras

Foram avaliadas 70 amostras de isolados (por análises bioquímicas de hidrólise na esculina e na produção de catalase, oxidase, urease e ácido a partir de arabinose, celobiose, lactose, salicina, sacarose, manitol e sorbitol) contendo $\boldsymbol{A}$. suis congelados em sangue de carneiro estéril. As amostras provenientes de plantéis suínos norte-americanos foram submetidas ao Laboratório de Diagnóstico Veterinário (Veterinary Diagnostic Laboratoy -VDL) da Universidade de Minnesota (University of Minnesota, Saint Paul) entre os anos de 2005 e 2008. Esses isolados foram originados de plantéis suínos dos Estados de Illinois, IL (10), Iowa, IA (6), Kansas KS (1), Minnesota, MN (26), Missouri,
MO (3), Carolina do Norte, NC (9), Oklahoma, OK (3), Dakota do Sul, SD (2) e Tennessee, TN (5). Para controle, foram utilizadas quatro cepas referências - ATCC (American Type Culture Collection).

Cultivo de cepas de $\boldsymbol{A}$. suis e extração de DNA

Cepas de A. suis foram cultivadas em meio sólido e ágar sangue de carneiro a 5\% por 24 horas, com temperatura de $37^{\circ} \mathrm{C}$, em meio aeróbio com $5 \%$ de $\mathrm{CO}_{2}$. Após o crescimento, duas colônias de bactérias foram adicionadas a dois microtubos contendo $300 \mu \mathrm{L}$ da solução tampão PBS (50mM de fosfato de potássio e $150 \mathrm{mM}$ de NaCl; $\mathrm{pH} 7,2$ ). Em seguida, os microtubos foram centrifugados por três minutos, a 3000rpm, com temperatura ambiente. O sobrenadante foi descartado, e o pellet foi utilizado para extração de DNA. Foram extraídos DNA das amostras utilizando o reagente Prepman (PrepMan Ultra sample preparation reagentApplied Biosystems, USA), de acordo com instruções do fabricante.

Reação em cadeia da polimerase-multiplex (PCRmultiplex)

PCR-multiplex foram realizadas para detectar genes codificadores das toxinas ApxI e ApxII como previamente descrito por RAYAMAJHI et al. (2005).

A solução (mix) para realização da PCRmultiplex foi preparada com os seguintes componentes: $25 \mu$ lde mastermix (Mastermix $^{\mathrm{R}}$ - Qiagen multiplex PCR KIT HotStarTaq DNA Polymerase, Multiplex PCR Buffer: $6 \mathrm{mM} \mathrm{MgCl}_{2}$ e dNTP Mix); $5 \mu \mathrm{l}$ de cada iniciador $(20 \mu \mathrm{M})$. Para amplificar o gene ApxI, foram utilizados os iniciadores APXIAF (ATC GAAGTACAT CGC TCG GA); APXIAR (CGC TAA TGC TAC GAC CGAAC); APXIBF(TTATCGCACTACCGGCAC TT)eAPXIBR (TGCAGT CAC CGA TTC CAC TA); para amplificar o gene ApxII, foram utilizados os iniciadores APXIIF (GCAATC AGT CCA TTG GCG TT) e APXIIR (CGT AAC ACC AGC AAC GAT TA). Além desses dois genes, foi amplificado o gene ApxIII, sendo utilizados os iniciadores APXIIIF (GCAATC AGT CCATTG GCG TT) e APXIIIR (GAC GAG CAT CAT AGC CAT TC). Todos esses iniciadores foram previamente descritos por RAYAMAJHI et al. (2005). Por fim, utilizou-se 50ng do DNA a ser testado e o volume final foi ajustado para $50 \mu \mathrm{L}$ com água ultrapura previamente tratada com DNase.

Controles foram feitos com as mesmas soluções e reagentes da PCR-multiplex, porém sem a presença do molde de DNA (controle negativo) e com 50ng DNA extraído de App dos sorotipos 1, 2, 4 e 6, e cada amostra correspondeu a um microtubo separado. As condições de reação foram: desnaturação inicial a 
$95^{\circ} \mathrm{C}$ por 15 minutos, seguida de 30 ciclos de $94^{\circ} \mathrm{C}$ por 30 segundos, $60^{\circ} \mathrm{C}$ por 1 minuto e $72^{\circ} \mathrm{C}$ por três minutos, e um passo final de extensão de $72^{\circ} \mathrm{C}$ por 10 minutos.

\section{Eletroforese em gel de agarose}

Os produtos da PCR-multiplex foram resolvidos em eletroforese, em gel de agarose $1 \%$, submetido a uma voltagem de $110 \mathrm{~V}$ por aproximadamente duas horas. Este foi visualizado e fotografadoem um transluminador (BioDoc- IT Imaging System ultraviolet light câmera, UPV). Os dados foram analisados pela presença ou ausência de bandas.

Extração e padronização das concentrações de proteína celular total.

Cepas de bactérias foram cultivadas em meio sólido, agar sangue de carneiro a $5 \%$ e posteriormente incubadas por 24 horas, com temperatura de $37^{\circ} \mathrm{C}$, em meio aeróbio com $5 \%$ de $\mathrm{CO}_{2}$ A extração proteica foi feita conforme o método descrito por OLIVEIRA \& PIJOAN, C. (2004), com pequenas modificações. As concentrações de proteínas extraídas foram determinadas por espectrofotometria, em um espectrofotômetro (BioPhotometer, Eppendorf model 613124242, German, a 280nm. Em seguida, as concentrações foram padronizadas para $1 \mathrm{mg} \mu \mathrm{L}^{-1}$.

Eletroforese em gel de poliacrilamida desnaturante SDS (SDS-PAGE)

Amostras de proteínas extraídas anteriormente foram submetidas à eletroforese em gel de poliacrilamida sob condições desnaturantes (SDSPAGE) $12 \%$. As condições da eletroforese foram as seguintes: $25 \mathrm{~mA}$ por 30 minutos e $35 \mathrm{~mA}$ por 4 horas e
10 minutos. A coloração do gel ocorreu em solução contendo ácido acético $10 \%$ (v/v), álcool metílico $40 \%$ (v/v) e Coomassie Brilliant Blue R-250 1\%, durante uma noite. O gel foi descorado em solução contendo $10 \%$ (v/v) de ácido acético e $40 \%$ (v/v) de álcool metílico. A análise do perfil de bandas ocorreu com auxílio do software BioNumerics, versão 2.5 (Applied Maths, Kortijk,Belgium), o qual detectou a presença ou ausência de bandas fazendo a comparação entre cada amostra.

\section{RESULTADOS E DISCUSSÃO}

Os resultados observados no gel de agarose $1 \%$ para avaliação da PCR-multiplex demonstram a amplificação de três bandas para os quatro sorotipos de App (Figura 1). No sorotipo 1, as bandas apresentavam tamanhos aproximados de $723 \mathrm{pb}$ (ApxIa), 811pb (ApxIb) e 965pb (ApxII). Entretanto, os sorotipo 2, 4 e 6 mostraram bandas com tamanhos próximos de 396pb (ApxIII), 965pb (ApxII) e 811pb (ApxIB). Esses achados concordam com os experimentos feitos por RAYAMAJHI et al. (2005). Conforme também pode ser observado na figura 1 , todos os isolados de A. suis foram positivos para a presença de genes codificadores das toxinas ApxI e ApxII, pois apresentam as bandas com tamanhos aproximados de $723 \mathrm{pb}, 811 \mathrm{pb}$ e $965 \mathrm{pb}$, que caracterizam, respectivamente, as toxinas ApxIa, ApxIb e ApxII (RAYAMAJHI et al., 2005). Assim, essas descobertas indicam uma similaridade entre as toxinas ApxI e ApxII produzidas por App, com toxinas hemolíticas/ citotóxicas produzidas por $\boldsymbol{A}$. suis, o que apoia a ideia de Kamp (1994). Contudo, não houve amplificação do

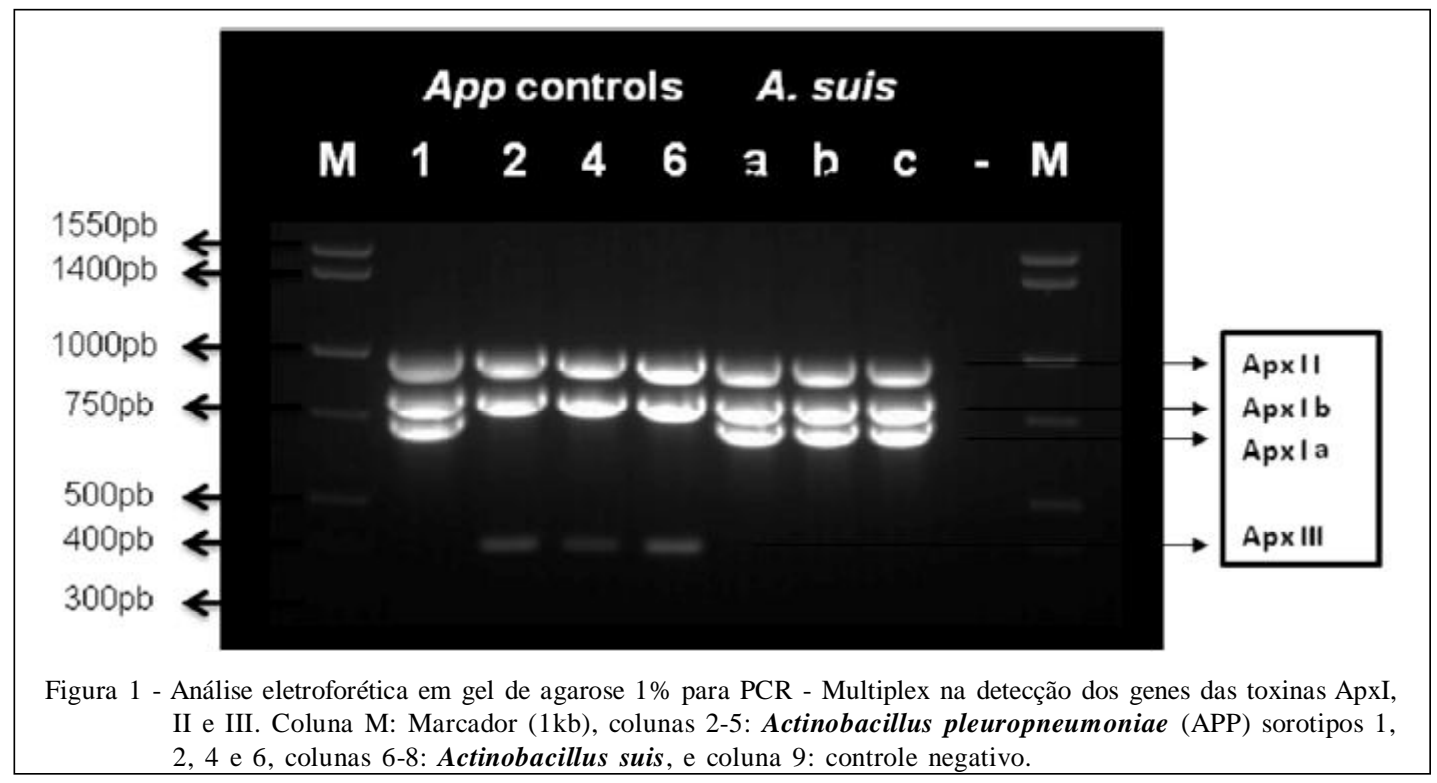

Ciência Rural, v.40, n.9, set, 2010. 
gene codificador da toxina ApxIII. Atualmente, há necessidade de descobertas de novas formas diagnósticas capazes de diferenciar as duas doenças de maneira rápida e sensível. Até hoje, a história clínica e as lesões são utilizadas para o diagnóstico pressuposto de pleuropneumonia, o qual é facilitado pela suspeita de surtos agudos da enfermidade. Nesses casos, o diagnóstico final é obtido pela observação das lesões características, além do isolamento do agente. Em casos crônicos, a identificação de App é difícil (NICOLET, 1992; STEVENSON, 1998). São necessários diagnósticos diferenciais de infecções causadas por A. suis e Salmonella choleraesuis ou a combinação de outros agentes, como Mycoplasma hyopneumoniae, Pasteurella multocida $e$ Mycoplasma hyorhinis; cujas lesões decorrentes são similares à pleuropneumonia (STEVENSON, 1998). Dessa forma, ApxIII poderia contribuir no diagnóstico diferencial, pois os resultados indicam essa toxina e seu gene como marcadores moleculares capazes de diferenciar infecções provocadas por $\boldsymbol{A}$. suis daquelas causadas por App dos sorotipos 2, 4 e 6 .

A análise de proteínas totais realizada com 70 amostras diferentes de $\boldsymbol{A}$. suis foi obtida por meio de SDS-PAGE. A figura 2 expõe o perfil de bandas de 13 das 70 amostras avaliadas pelo programa BioNumerics, versão 2.5, o qual identificou e comparou as bandas obtidas, comprovando que todas essas amostras apresentavam as mesmas bandas. Dessa forma, este trabalho confirmou que os isolados de A. suis, circulantes em rebanhos norte-americanos, são altamente clonais, ou seja, similares entre diferentes indivíduos da mesma espécie. Assim, essas bactérias podem produzir proteínas similares, tais como toxinas e outras proteínas celulares.

Este é o primeiro estudo que caracteriza de modo compreensível o $\boldsymbol{A}$. suis relacionado com doença nos Estados Unidos, o que, em associação com parâmetros, como campo de isolamento da bactéria, grau de lesão no animal e origem desta, ausência de lesões por outros patógenos, prevalência no rebanho e ano de isolamento, pode representar a oportunidade para a criação de uma vacina universal para $\boldsymbol{A}$. suis. Destaca-se que, até o momento, não existem vacinas comerciais contra $A$. suis, e a vacinação é uma opção válida para o controle, em comparação com os tratamentos intensivos com antibióticos, que podem interferir na colonização do trato respiratório por membros na flora normal, além de ser mais barata a prevenção com a vacinação do que o tratamento com antibiótico (OLIVEIRA, 2007). As informações referentes à eficácia de vacinas autógenas são escassas. Contudo, como demonstrado neste trabalho, a variabilidade fenotípica de A. suis é limitada, aumentando as chances de que uma vacina universal produza proteção contra isolados de campo.

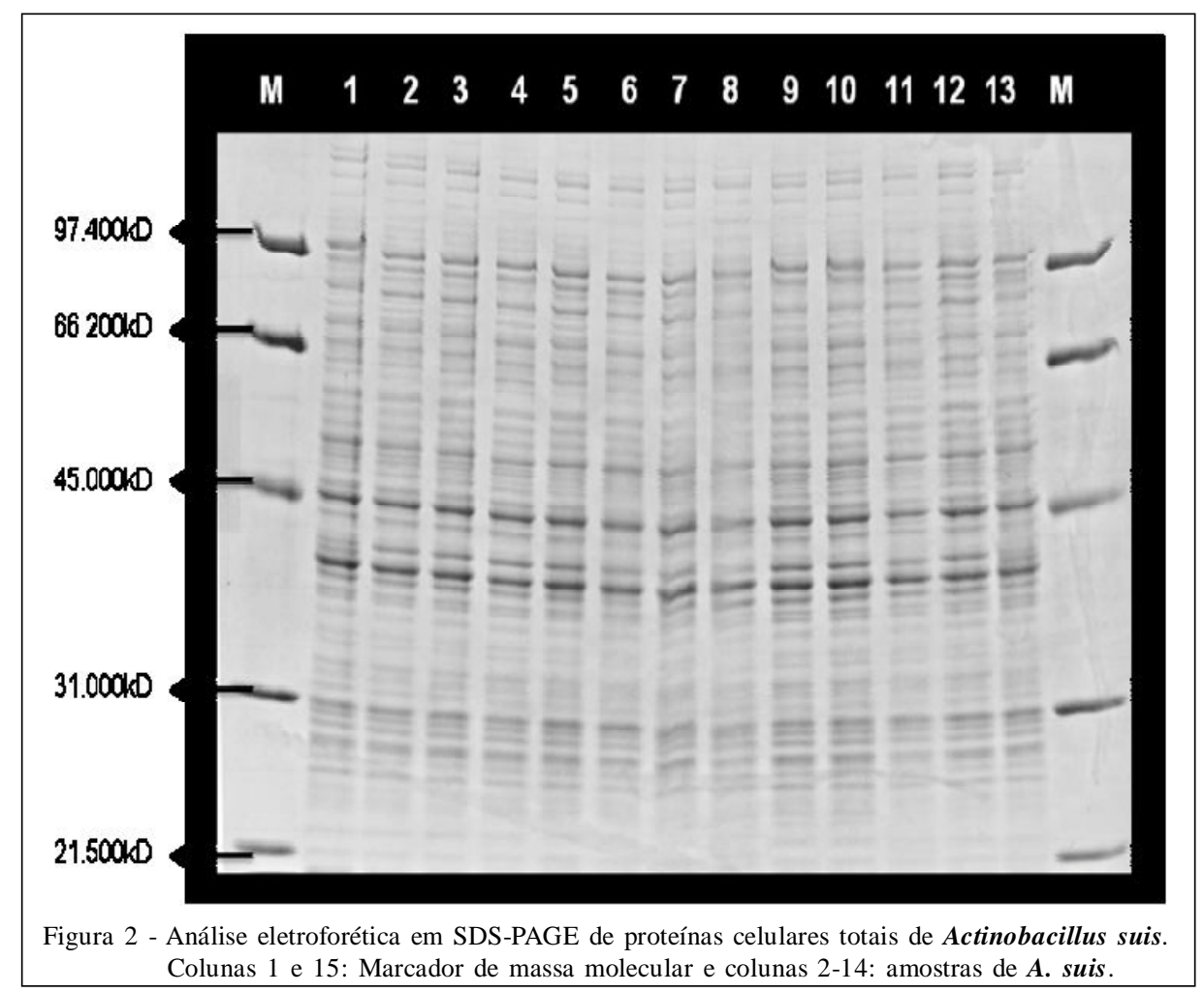

Ciência Rural, v.40, n.9, set, 2010. 
Entretanto, mais informações sobre a associação entre virulência, antígenos (cápsula, proteínas de membrana, toxinas) e imunidade protetora são necessárias para o desenvolvimento de vacinas eficazes.

\section{CONCLUSÃO}

As cepas de $\boldsymbol{A}$. suis presentes nos plantéis de suínos norte-americanos podem ser clonais, por apresentarem perfis proteicos idênticos, além disso, todas possuem genes semelhantes ou idênticos aos genes que codificam as proteínas ApxI e ApxII, e não possuem o gene semelhante ou idêntico codificador da toxina ApxIII. Todos esses genes foram encontrados em App.

O gene da proteína ApxIII pode ser utilizado como marcador para desenvolver um diagnóstico diferencial entre infecções causadas por $\boldsymbol{A}$. suis e infecções causadas por App dos sorotipos 2, 4 e 6.

\section{REFERÊNCIAS}

BURROWS, L.L.; LO, R.Y. Molecular characterization of an RTX toxin determinant from Actinobacillus suis. Infection and Immunity, v.60, n.6, p.2166-2173, 1992. Disponível em: <http://www.ncbi.nlm.nih.gov/pmc/articles/PMC257139/ ?report=abstract $>$. Acesso em: 20 nov. 2008.

COLLARES, R.M. Análise molecular dos genes para as toxinas de $A$. pleuropneumoniae em isolados de campo. 2000. 85f. Dissertação (Mestrado em Ciências Veterinárias) Curso de Pós-graduação em Ciências Veterinárias, Universidade Federal do Rio Grande do Sul,RS. Disponível em: <http:// www.scielo.br/scielo.php? script=sci_arttext\&pid=S010384782004000400057>. Acesso em: 16 set. 2008.

GEIGER, J. et al. Lowering the prevalence of Actinobacillus suis. In: International Pig Veterinary Society Congress, 20., 2008, Durban, South Africa. Proceedings... Dublin, 2008. AASV Swine information CD- ROM catalog. Disponível em: <http:/ /www.pigprogress.net/public/file/IPVS oral\%20presentations/ B a c teria $1 \% 20$ Diseases/Actinobacillus/ L o w e r i n g \% $20 \mathrm{th}$ e $20 \mathrm{pr}$ e vale 1 c e $\% 20$ of $\% 20$ Actinobacillus $\% 20$ suis.pdf $>$. Acesso em: 10 nov. 2009 .

GRAM, T. et al. An A. pleuropneumoniae PCR typing system based on the apx and omla genes-evaluation of isolates from lungs and tonsils of pigs. Veterinary Microbiology, v.75, p.43-57, 2000. Disponível em: <http://www.sciencedirect.com/ science?_ob=ArticleURL\&_udi=B 6T D 6-40J1DVM$5 \&$ \& ser $=687360 \&$ \&coverDate $=07 \% 2$ F0 $3 \% 2$ F $2000 \&$ _alid $=1222175595 \& \_r d o c=1 \& \_f m t=h i g h \& \_o r i g=s e a r c h \& \_c d i=5190$ \&_sort $=$ r\&_st $=4$ \&_docanchor $=\&$ _ct $=11$ \&_acct $=\mathrm{C} 000037900 \&$. version $=1 \& \_u r l$ Version $=0 \& \_u$ serid $=687360 \& \mathrm{md} 5=6 \mathrm{f} 6 \mathrm{c} 0 \mathrm{ff} 98 \mathrm{ab}$ 74766008ef05eac3eeff1>. Acesso em: 17 out. 2009. doi:10.1016/ S0378-1135(00)00206-6.

KAMP, E.M. et al. Production of Apx toxins by field strains of Actinobacillus pleuropneumoniae and Actinobacillus suis. Infection and Immunit, v.62, n.9, p.4063-4065, 1994.
Disponível em: <http://www.ncbi.nlm.nih.gov/pmc/articles/ PMC303069/>. Acesso em: 10 jun. 2009.

NICOLET, J. Actinobacillus pleuropneumoniae. In: LEMAN, A.D. et al. Diseases of swine. Iowa: Wolfe, 1992. Cap.31, p.401-408.

OJHA, S. et al. Characterization of colonization-deficient mutants of Actinobacillus suis. Veterinary Microbiology, v.140, p.122130, 2010. Disponível em: <http://www.sciencedirect.com/ science?_ob=ArticleURL\&_udi=B 6T D 6-4 WR 66FD$7 \&$ _user $=687360 \&$ _coverDate $=01 \% 2$ F $06 \% 2$ F 2010

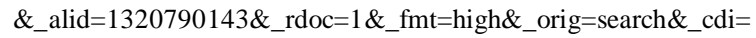
5190\&_sort=r\&_docanchor $=\& v i e w=c \& \_c t=31 \& \_a c c t=C 000037900 \&$ version $=1 \&$ _urlVersion $=0 \&$ _userid $=687360 \& \mathrm{~m}$ d5=2f64d19a99a19b7246a707167a822cbd>. Acesso em: 20 abr. 2010. doi:10.1016/j.vetmic.2009.07.014.

OLIVEIRA, S. Actinobacillus suis diagnostics, epidemiology and control - on the path from good to great. In: AMERICAN ASSOCIATION OF SWINE VETERINARIANS ANNUAL MEETING, 38., 2007, Orlando, Florida, USA. Proceedings... AASV, 2007. p.371-376.

OLIVEIRA, S.; PIJOAN, C. Computer-based analysis of Haemophilus parasuis protein fingerprints. Canadian Journal Veterinary Reserch, v.68, n.1, p.71-75, 2004. Disponível em: <http://www.ncbi.nlm.nih.gov/pmc/articles/ PMC1142133/>. Acesso em: 23 jun. 2009.

OSTAAIJEN, J.V. et al. Actinobacillus suis strains isolated from healthy and diseased swine are clonal and carry $a p x I C A B D D_{\text {varsuis }}$ and apxIICAvar.suis toxin genes. Journal of Clinical Microbiology, v.35, n.5, p.1131-1137, 1997. Disponível em: <http://jcm.asm.org/ cgi/content/abstract/35/5/1131>. Acesso em: 15 ago. 2009.

RAYAMAJHI, N. et al. Development and use of multiplex polymerase chain reaction assay based on Apx toxin genes for genotyping of Actinobacillus pleuropneumoniae isolates. Journal of Veterinary Diagnostic and Investigation, v.17 p.359-362, 2005. Disponível em: <http://www.ncbi.nlm.nih.gov/ sites/entrez>. Acesso em: 15 ago. 2009.

SCHALLER, A. et al. Characterization of Apx IVA, a new RTX determinant of A. pleuropneumoniae. Microbiology, v.145, p.2015-2016, 1999.

SCHALlER, A. et al. Apx toxins in Pasteurellaceae species from animals. Veterinary Microbiology, v.74, p.47-62, 2000. Disponível em: <http://www.sciencedirect.com/ science?_ob=ArticleURL\&_udi=B6TD6-409631 T$7 \&$ \&user $=687360 \&$ _coverDate $=06 \% 2 \mathrm{~F} 12 \% 2 \mathrm{~F} 2000 \& \_$rdoc $=1 \&$ _ $\mathrm{fmt}=$ high \&_orig $=$ search \&_sort $=\mathrm{d} \& \_$docanchor $=\& \mathrm{view}=\mathrm{c} \&$ _searchStrId=1222238480\&_rerunOrigin $=$ google\&_acct $=\mathrm{C}$ 000037900 \&_version $=1$ \&_urlVersion $=0 \& \_u s e r i d=687360 \& \mathrm{md} 5=$ 3e0345257e1ab85738a1045ddb38013a>. Acesso em: 20 out. 2009. doi:10.1016/S0378-1135(00)00204-2.

SLAVIC, D. et al. Comparative pathogenicity of different Actinobacillus suis $\mathrm{O} / \mathrm{K}$ serotypes. Canadian Journal of Veterinary Research, v.64, p.81-87, 2000. Disponível em: <http://www.ncbi.nlm.nih.gov/pmc/articles/PMC1189589/>. Acesso em: 20 out. 2009.

STEVENSON, G.W. Bacterial pneumonia in swine. In: INTERNATIONAL PIG VETERINARY SOCIETY CONGRESS, 1998, Birmingham. Proceedings... Birmingham: International Pig Veterinary Society, 1998. p.11-20. 